\title{
Interaction Study of the Male Specific Lethal (MSL) Complex and Trans-Acting Dosage Effects in Metafemales of Drosophila melanogaster
}

\author{
X. Sun J.A. Birchler \\ Division of Biological Sciences, University of Missouri, Columbia, Mo., USA
}

\author{
Key Words \\ Dosage compensation - Histone acetylation - Metafemales • \\ MSL2
}

\begin{abstract}
The effect of ectopic expression of male specific lethal 2 ( $m s / 2$ ) on chromatin modification and gene expression was studied in Drosophila diploid females and metafemales $(3 \mathrm{X} ; 2 \mathrm{~A})$. Results show that ectopic expression of MSL2 in transgenic $m s / 2$ females and metafemales sequesters the MOF histone acetylase to the $\mathrm{X}$, which occurs concordantly with an increase of histone acetylation. Gene expression studies indicate that the X-linked genes are not affected by direct targeting of the MSL complex and the resulting increased H4Lys16 acetylation on the X chromosomes, suggesting one function of the MSL complex is to nullify the effect of a high level of histone acetylation. These results are not consistent with the hypothesis that the presence of the MSL complex conditions a twofold upregulation. Autosomal gene expression is generally decreased in ectopically expressed MSL2 females, which correlates with the reduced autosomal histone acetylation. Metafemales show dosage compensation of X-linked genes with some autosomal reductions in expression. Interestingly, in metafemales with ectopically expressed MSL2, the autosomal expression is returned to a more normal level. There is a lower autosomal level of histone acetylation compared to the normal metafemales, suggesting a nullifying effect on the negative dosage effect of the $\mathrm{X}$ chromosome as previously hypothesized to occur in normal males.

Copyright $\odot 2009$ S. Karger AG, Basel
\end{abstract}

(C) 2009 S. Karger AG, Basel

$1424-8581 / 09 / 1244-0298 \$ 26.00 / 0$

Fax +4161306 1234

E-Mail karger@karger.ch

www.karger.com
Accessible online at:

www.karger.com/cgr
Dosage compensation is the phenomenon of equalizing gene expression from chromosomes of different copy numbers [Muller, 1932]. Sex chromosomal dosage compensation between the single $\mathrm{X}$ chromosome in males and the two X chromosomes in females has been mostly studied in Drosophila, mammals and nematodes. It has recently been demonstrated that a common feature of dosage compensation occurs among these organisms, namely, that the single $\mathrm{X}$ chromosome is up-regulated about twofold [Birchler et al., 2006; Gupta et al., 2006; Nguyen and Disteche, 2006]. In Drosophila, the twofold up-regulation of the male X compared to one of the $2 \mathrm{X}$ chromosomes in females has been postulated to rely on the Male Specific Lethal (MSL) complex [Belote and Lucchesi, 1980; Kuroda et al., 1991]. This complex consists of the protein products of 6 genes ( $m s l 1, m s l 2, m s l 3$, mle, mof and JIL1) and 2 non-coding roX RNAs [Kuroda et al., 1991; Meller et al., 1997; Jin et al., 1999, 2000; Wang et al., 2001]. This explanation is based on the fact that the male X chromosome associates with the MSL complex, and the presence of the complex on the $\mathrm{X}$ chromosome brings a histone acetyltransferase (MOF) and kinase (JIL1) to the X, which causes elevated levels of $\mathrm{H} 4$ acetylation (H4LysAc16) and H3 phosphorylation [Turner et al., 1992; Bone et al., 1994; Hilfiker et al., 1997; Jin et al., 1999, 2000; Wang et al., 2001]. Because histone acetylation is usually associated with higher levels of gene expression [Brownell and Allis, 1996; Wolffe and Pruss, 1996; Grunstein, 1997], the MSL complex has been hypothesized by others to be responsible for $\mathrm{X}$ chromosomal dosage compensation. However, gene expression studies on 
the msl mutants, including mle (maleless) and mof, and other circumstances in which the complex is destroyed, have demonstrated that most X-linked genes remain compensated and many autosomal genes are increased in expression [Hiebert and Birchler, 1994; Bhadra et al., 1999, 2000; Pal Bhadra et al., 2005, 2006]. Moreover, dosage compensation occurs in metafemales with $3 \mathrm{X}$ chromosomes [Margolis, 1934; Stern, 1960; Lucchesi et al., 1974; Birchler et al., 1989] as well as in the male germline [Gupta et al., 2006]. There is no MSL complex in metafemales (present study) or the male germline [Rastelli and Kuroda, 1998], suggesting that the MSL complex and associated histone acetylation might not fully explain dosage compensation. We have previously suggested that an inverse dosage effect produced by the $\mathrm{X}$ to autosomal genomic imbalance could account for the proper modulation of $\mathrm{X}$ expression in various genotypes exhibiting compensation [Hiebert and Birchler, 1994; Birchler, 1996; Birchler et al., 2006].

The inverse dosage effect is thought to result from stoichiometric changes of members of multi-subunit molecular regulatory complexes - a formulation referred to as the gene balance hypothesis [Birchler and Newton, 1981; Birchler et al., 2001, 2005; Birchler and Veitia, 2007; Veitia et al., 2008]. One major aspect of the evidence for this concept is that alterations of gene expression are more extensive in aneuploids than in ploidy series [Guo and Birchler, 1994; Guo et al., 1996]. Individual genes responsible for these types of effects are primarily transcription factors and members of signal transduction cascades [Birchler et al., 2001]. As a result of the prevalence of an inverse dosage effect, dosage compensation occurs for many genes in any substantial aneuploid and genes elsewhere in the genome are affected in trans including for human trisomies [Birchler, 1979, 1981; Devlin et al., 1982; 1988; Birchler et al., 1990; Guo and Birchler, 1994; Ait Yahya-Graison et al., 2007; Altug-Teber et al., 2007]. Dosage compensation results from the simultaneous regulatory inverse dosage effect that cancels a structural gene dosage effect of target loci on the same chromosome [Birchler, 1981; Birchler et al., 1990]. The responsible mechanism has likely been co-opted and enhanced for sex chromosome dosage compensation.

That the X-linked genes in male $m s l$ mutants are primarily compensated implies that these genes do not respond to the increased level of histone acetylation on the $\mathrm{X}$ chromosome in normal males. Previous evidence from our laboratory suggested that the MSL complex involves a counteraction of histone acetylation [Bhadra et al., 1999; Pal Bhadra et al., 2005]. Indeed, in ISWI mutant embryos, this counteraction appears to be eliminated, allowing Xlinked genes to respond to the high level of histone acetylation [Pal Bhadra et al., 2005]. In contrast, the autosomal gene expression in $m s l$ mutants is increased, which parallels the increase of histone acetylation on the autosomes caused by redistribution of MOF and JIL1 kinase [Bhadra et al., 1999, 2000; Pal Bhadra et al., 2005]. Furthermore, in circumstances in which SXL is eliminated in females, thus allowing MSL association with the $\mathrm{X}$ chromosomes, we find no generalized change in gene expression for the $\mathrm{X}$ chromosomes, but a reduction for the autosomes [Bhadra et al., 2000; Pal Bhadra et al., 2005, 2006]. This reduced gene expression is consistent with the depletion of histone acetylation on the autosomes in the presence of abnormally expressed MSL2, a critical component required for the localization of the MSL complex to the X. The failure of the $\mathrm{X}$ chromosomes to increase in expression is consistent with a counteraction effect of the MSL complex or associated activities. From these studies, it is postulated that the MSL complex in normal males overrides the impact of histone acetylation on gene expression but allows a twofold up-regulation resulting from genomic imbalance to achieve the proper level of dosage compensation. In addition, it also mutes the global inverse effect on the autosomes by sequestering MOF to the $\mathrm{X}$ chromosome to equalize the autosomal gene expression between the sexes [Hiebert and Birchler, 1994; Bhadra et al., 1999, 2000; Pal Bhadra et al., 2005].

As mentioned above, dosage compensation has been observed in metafemales (3X;2A). The expression of each gene copy present must be reduced to two thirds so that the total amount from the $3 \mathrm{Xs}$ is equivalent to a normal female. Autosomal expression in these flies is also reduced to approximately two thirds of normal, presumably as a negative dosage effect of the $\mathrm{X}$ chromosomal copy number [Birchler et al., 1989; Birchler, 1992]. In order to more extensively study the interactions of the MSL complex with genomic imbalance, and to examine their influence on gene expression, the present study explored histone acetylation and gene expression in diploid males, females and metafemales with ectopically expressed MSL2.

Our results demonstrate that the expression of X-linked genes is not affected by the targeting of the MSL complex. Autosomal gene expression is generally decreased in ectopically expressing MSL2 females and nontransgenic metafemales. In ectopically expressing MSL2 metafemales autosomal gene expression is returned to more normal levels, suggesting that the negative dosage effect of the $\mathrm{X}$ chromosome is apparently ameliorated in metafemales as previously suggested to occur in normal males. 


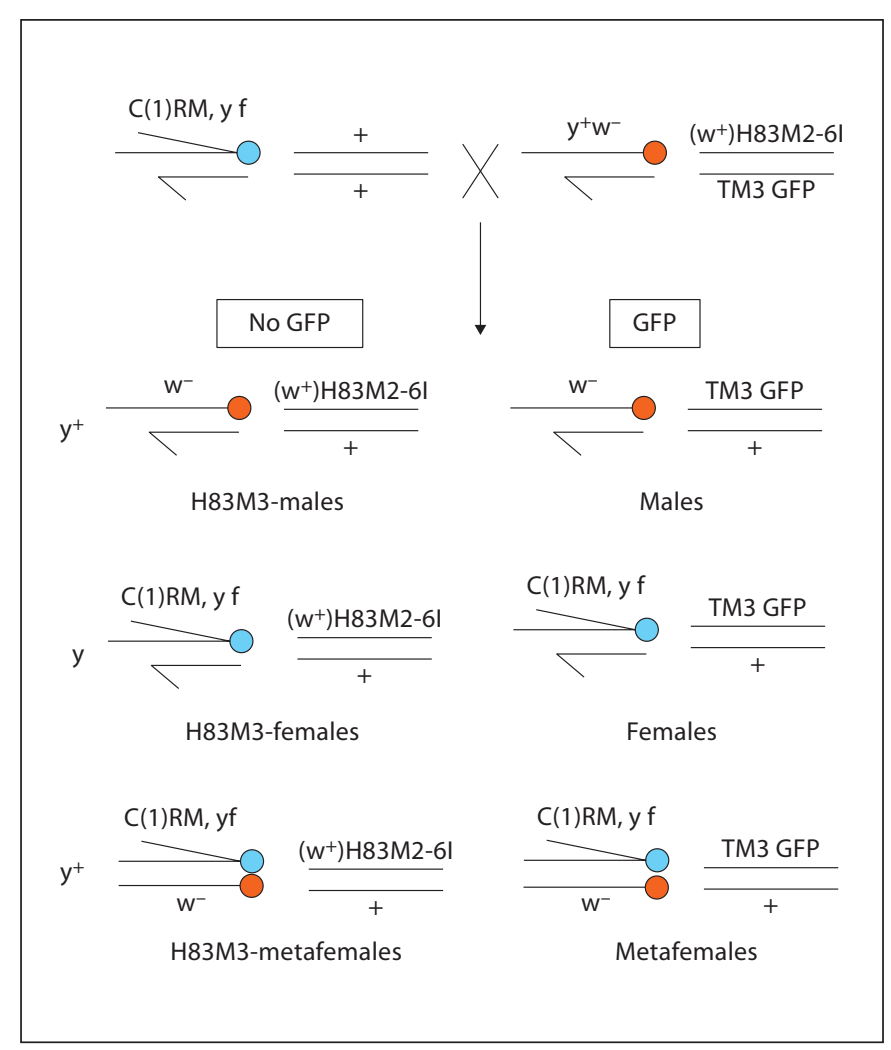

Fig. 1. Genetic cross to generate transgenic $m s l 2$ females and metafemales. 6 genotypes of progeny are produced from the cross of $w-/ Y$; +/+; (w+)H83M2-6I/TM3,GFP males with C(1)RM, $y f / Y$ (with attached $X$ chromosomes) females. These genotypes include: 1$) w-/ Y ;+/+;(w+) H 83 M 2-6 I /+$ (males with transgene), 2) $w-/ Y$; +/+; TM3,GFP/+ (males without transgene), 3) C(1)RM, $y \mathrm{f} / \mathrm{Y}$; +/+; $(w+) H 83 M 2-6 I /+$ (females with transgene), 4) C(1)RM, y $f / Y$; +/+; TM3,GFP/+ (females without transgene), 5) C(1)RM, y $\mathrm{f} / \mathrm{w}-;+\mathrm{+}+; \quad(w+) H 83 \mathrm{M} 2-6 \mathrm{I} /+$ (metafemales with transgene), 6) C(1)RM, $y$ f/w-; +/+; TM3,GFP/+ (metafemales without transgene). They are referred to as H83M2-males, normal males, H83M2-females, normal females, H83M2-metafemales and normal metafemales, respectively.

\section{Materials and Methods}

\section{Drosophila Stocks and Crosses}

Mutations, genes and chromosomal balancers are described in Flybase (http://flybase.bio.indiana.edu). Flies were cultured on cornmeal dextrose medium at $25^{\circ} \mathrm{C}$. To produce ectopically expressing MSL2 females and metafemales, the transgene $\left[\left(w^{+}\right) H 83 M 2-6 I\right]$, a P-element msl2 construct with a mini-white reporter gene [Kelley et al., 1995], was introduced by the following crosses. The genetic markers $S b$, GFP and $w+$ were used to classify the different types of progeny. In brief, the $w-/ Y$; msl1/CyO; $(w+) H 83 M 2-6 I$ males were crossed with $w-/ w-;+/+; S b / T M 3, G F P$ females. The male progeny $w-/ Y$; $+/ C y O ;(w+) H 83 M 2-6 I / T M 3$, GFP were selected for backcross to $w-/ w-;+/+; S b / T M 3$, GFP fe- males. Male $w-/ Y ;+/+;(w+) H 83 M 2-6 I / T M 3$, GFP progeny from the backcross were selected and crossed to $C(1) R M, y f / Y$ females (with attached X chromosomes). From this type of cross, 6 classes of progeny are generated (fig. 1). Each genotype of larvae was separated by sex, the presence or absence of GFP and mouth hook color to score for the $y$ mutation. The males, females and metafemales without $(w+)$ H83M2-6I were used as controls for Northern analysis and chromatin staining.

\section{RNA Preparation}

Total RNA was extracted using TRIZOL ${ }^{\circledR}$ Reagent (Invitrogen). Briefly, 6 genotypes of 3rd instar larvae were sorted in Ringer's solution $\left(3.6 \mathrm{mM} \mathrm{NaCl}, 54.3 \mathrm{mM} \mathrm{KCl}, 8.0 \mathrm{mM} \mathrm{CaCl}_{2}\right.$, and 28.3 $\mathrm{mM} \mathrm{MgCl}_{2}$ ), collected into $1.5 \mathrm{ml}$ microcentrifuge tubes and frozen at $-80^{\circ} \mathrm{C}$. Larvae were homogenized in $1 \mathrm{ml}$ of TRIZOL per 40-50 larvae using a powered tissue homogenizer (Tekmar). After homogenization, the homogenate was centrifuged at $12,000 \mathrm{~g}$ for $10 \mathrm{~min}$ at $-4^{\circ} \mathrm{C}$ to remove debris. The supernatant was collected into a new centrifuge tube and then shaken vigorously for $15 \mathrm{~s}$ after the addition of $0.2 \mathrm{ml}$ chloroform. The mixture was centrifuged for $15 \mathrm{~min}$ at $12,000 \mathrm{~g}$ at $4^{\circ} \mathrm{C}$ and separated into 3 layers. The upper aqueous phase where RNA remained was collected. RNA was precipitated in $0.5 \mathrm{ml}$ of isopropyl alcohol and the pellet was washed once in $75 \%$ ethanol (in DEPC-treated water). The RNA pellet was dissolved in $100 \%$ formamide (deionized) and the RNA concentration was determined using a Nano-Drop hND1000 spectrophotometer. The isolated RNA was stored at $-80^{\circ} \mathrm{C}$ for Northern analysis.

\section{Northern Analysis}

Total RNA was separated on formaldehyde-agarose gels (1.5\%) [Sambrook and Russell, 2001] at $10 \mu \mathrm{g}$ per lane. Gels were run at approximately $50 \mathrm{~V}$ for $16 \mathrm{~h}$. The RNA was transferred onto Hybond-N nylon membrane (Amersham Bioscience) by the TurboBlotter ${ }^{\circledR}$ downward transfer system (Schleicher \& Schuell) using $20 \times$ SSC, and then UV cross-linked to the filter. Hybridizations were performed as described previously [Birchler et al., 1989]. Hybridized filters were washed 3 times at $75^{\circ} \mathrm{C}$ in washing buffer consisting of $0.2 \times$ SSC and $0.05 \%$ SDS. Filters were dried and subjected either to autoradiography with Kodak XAR film or to a Fujifilm Fluorescent Image Analyzer FLA-2000 (Fuji, Tokyo, Japan) to detect mRNA signals. The band intensity was measured using the Fujifilm Image Gauge V 3.3 program.

In this study, rRNA is used as a loading control for comparisons of gene transcripts between transgenic genotypes and their normal control. This is based on the assumption that equivalent amounts of total RNA from these compared genotypes are contributed from equal amounts of DNA. In order to test this assumption, we isolated total nucleic acid of each genotype and determined the total DNA/rRNA ratios according to the method described [Hiebert and Birchler, 1994]. Triplicate isolations of the different genotypes were subjected to electrophoresis at $1.5 \mu \mathrm{g} /$ lane in $1 \%$ agarose gel (as shown in fig. 2). In the same gel, a dilution series of nucleic acid was included to ensure that the amount in the $1.5 \mu \mathrm{g}$ per lane falls within the linear range. Separate DNaseI and RNase A digestions confirmed the respective rRNA and genomic DNA positions on the ethidium-stained gel (not shown). The results reveal that there were no significant differences on DNA/28S rRNA ratios between the transgenic $m s l 2$ genotypes with their respective normal genotypes or between meta- 
females and normal females, indicating that it is valid to use rRNA as a loading control for comparisons and that the Northern comparisons are equivalent to 'per DNA' comparisons.

\section{Probe Preparation}

Northern blots were probed with ${ }^{32} \mathrm{P}$-labeled antisense RNA probes. The probes were prepared with T3, T7 or SP6 RNA polymerase from the linearized cDNAs using MAXIscript $\mathrm{P}$ In vitro transcription kit (Ambion). cDNAs described below were linearized with the proper restriction endonucleases (from Promega or NEB) and purified with a DNA purification kit (Promega). Synthesized probes were purified by size exclusion RNase-free Sephadex G-50 spin columns described in the MAXIscript kit to remove the unincorporated nucleotides.

Gene expression measured in this study included 6 X-linked genes: yellow (3), vermilion (6), Sgs4 (3), G6pdh (5), 6Pgdh (6) and rudimentary (3), and 7 autosomal genes: $\alpha$-Gpdh (5), Adh (7), Su$\operatorname{var}(2-5)$ (3), $\beta$-tubulin (3), SevenUp (3), Rp49 (5) and $D d c$ (7). The number of replicate Northern blots for each gene is given in parentheses. All cDNA clones were from laboratory permanent stocks and have been described in previous publications [Hiebert and Birchler, 1994; Bhadra et al., 2000], except Suvar(2-5) cDNA, which encodes the HP1 protein and which was provided by Drs. Nicole Riddle and Sarah Elgin from Washington University (St. Louis). The choice of genes was guided by those used previously in studies of dosage compensation. The number of genes analyzed was limited by the ectopically expressing MSL2 metafemales of which only 36 were recovered over a 10 month collection period. The endogenous white gene could not be included because it was deleted in some genotypes and present on the H83M2-6I transgene.

\section{Data Analysis}

All statistical analyses were conducted using SAS (SAS Institute, 1988). Northern data (as a ratio of transcript/rRNA) were subjected to an analysis of variance using PROC GLM (SAS Institute, 1988). Differences between means were considered statistically significant at the level of $\mathrm{p} \leq 0.05$ based on paired t tests.

\section{Immunostaining of Chromosomes}

Polytene chromosomes from the third instar larvae were dissected, fixed, and processed for antibody staining according to the protocols as described by others [Kuroda et al., 1991; Hilfiker et al., 1994; Pal Bhadra et al., 1997]. In brief, salivary glands were dissected in $0.7 \% \mathrm{NaCl}$ and fixed in PBS solution containing $0.1 \%$ Triton X-100, 3.7\% formaldehyde on a siliconized coverslip for $1 \mathrm{~min}$, then in $50 \%$ acetic acid, $3.7 \%$ formaldehyde for $2-5 \mathrm{~min}$. The coverslip was picked up with the slide and inverted. The glands were squashed and the slides were placed in liquid nitrogen or on dry ice for the separation of the coverslip from the slide. After the removal of all coverslips, the slides were washed in PBS and blocked with PBT (PBS, 1\% BSA, 0.2\% Triton X-100, 0.02\% azide) for $30 \mathrm{~min}$. The primary antibody binding was performed at an appropriate dilution in $\mathrm{PBT}$ at $4^{\circ} \mathrm{C}$ overnight. The second antibody conjugated with fluorophore (diluted 1:100 to 1:200 in PBT) was performed at room temperature for $30 \mathrm{~min}$ to $3 \mathrm{~h}$. The slides were mounted with Vectashield mounting medium containing 4',6-diamidino-2-phenylindole (DAPI) (Vector Laboratory, Inc. Burlingame, CA) and examined with a Zeiss fluorescence microscope (Carl Zeiss, Inc, Oberkochen, Germany). The images were prepared using Adobe Photoshop 7.0 software.

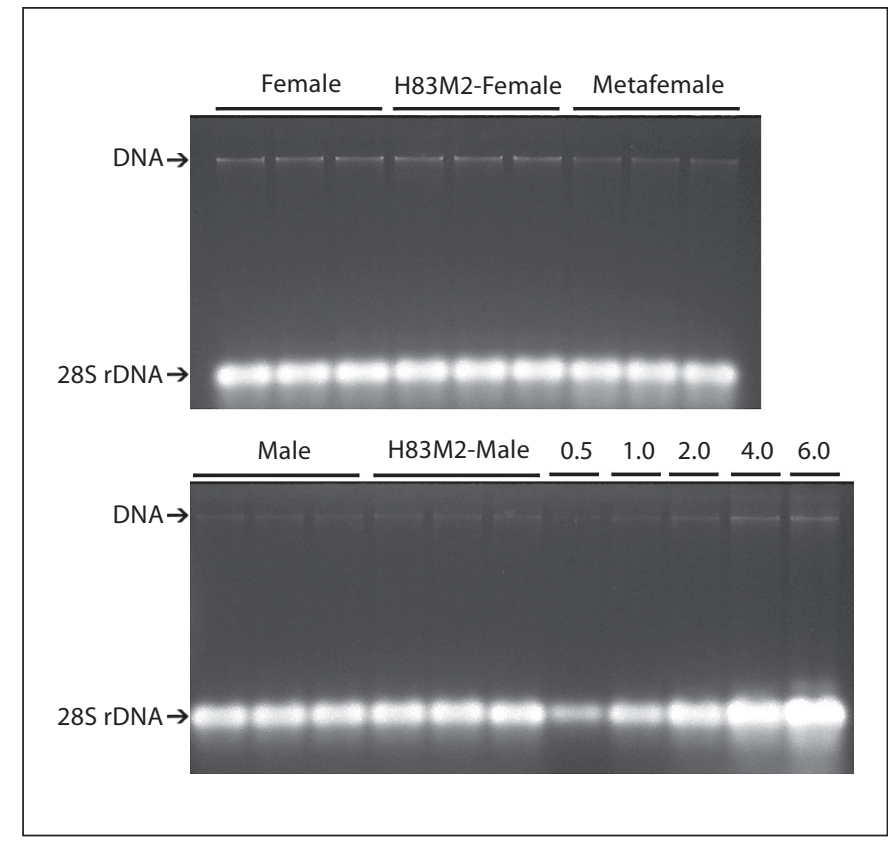

Fig. 2. Examination of DNA/rRNA ratios in males, females, H83M2-females and metafemales. Ethidium bromide-stained agarose gel images show the comparisons of DNA/rRNA ratios in the noted genotypes. Three replicates of total nucleic acid extraction are shown for each genotype. Each replicate is loaded with $1.5 \mu \mathrm{g} /$ lane. Gel bands labeled with DNA and 28S rRNA were confirmed by DNaseI and RNase A digestion. A dilution series shown to the right of the bottom image is from 0.5 to $6.0 \mu \mathrm{g}$. The samples in both top and bottom images were electrophoresed in the same gel.

\section{Results}

The MSL complex is comprised of 6 protein subunits, MSL1, MSL2, MSL3, MLE, MOF and JIL1, and at least 2 noncoding RNAs, roX1 and roX2 [Kuroda et al., 1991; Hilfiker et al., 1997; Meller et al., 1997; Jin et al., 1999, 2000; Wang et al., 2001]. Studies have shown that MSL2 is a critical component for the localization of the complex on the male $\mathrm{X}$, and also is required together with the other MSL proteins for the presence of the Lys16Ac isoform of histone H4 [Bone et al., 1994]. The $m s l 2$ gene has been reported to encode a ring finger protein. The $m s l 2$ gene is transcribed in both sexes but can not be translated in females [Kelley et al., 1995]. By circumventing the translational block, ectopic expression of MSL2 protein in females has been documented to target the $2 \mathrm{X}$ chromosomes and results in the appearance of the complex proteins (MSL2, MSL1, MLE) on the female Xs with a parallel enrichment of H4Ac16 [Kelley et al., 1995; Bhadra et al., 1999]. In this 
Fig. 3. Ectopic expression of MSL2 in females and metafemales. Immunofluoresence staining of polytene chromosomes with anti-MSL2 (green) from third instar male, female and metafemale larvae carrying the $(w+) H 83 M 2-6 I$ transgene. Nuclei were stained with DAPI in blue. The left upper image represents normal males and upper right the overexpressing MSL2 males. The lower left is ectopically expressing MSL2 females and lower right ectopically expressing MSL2 metafemales. The staining from normal males without $(w+) H 83 M 2-6 I$ was used as a comparison. The scale bar represents $15 \mu \mathrm{m}$.
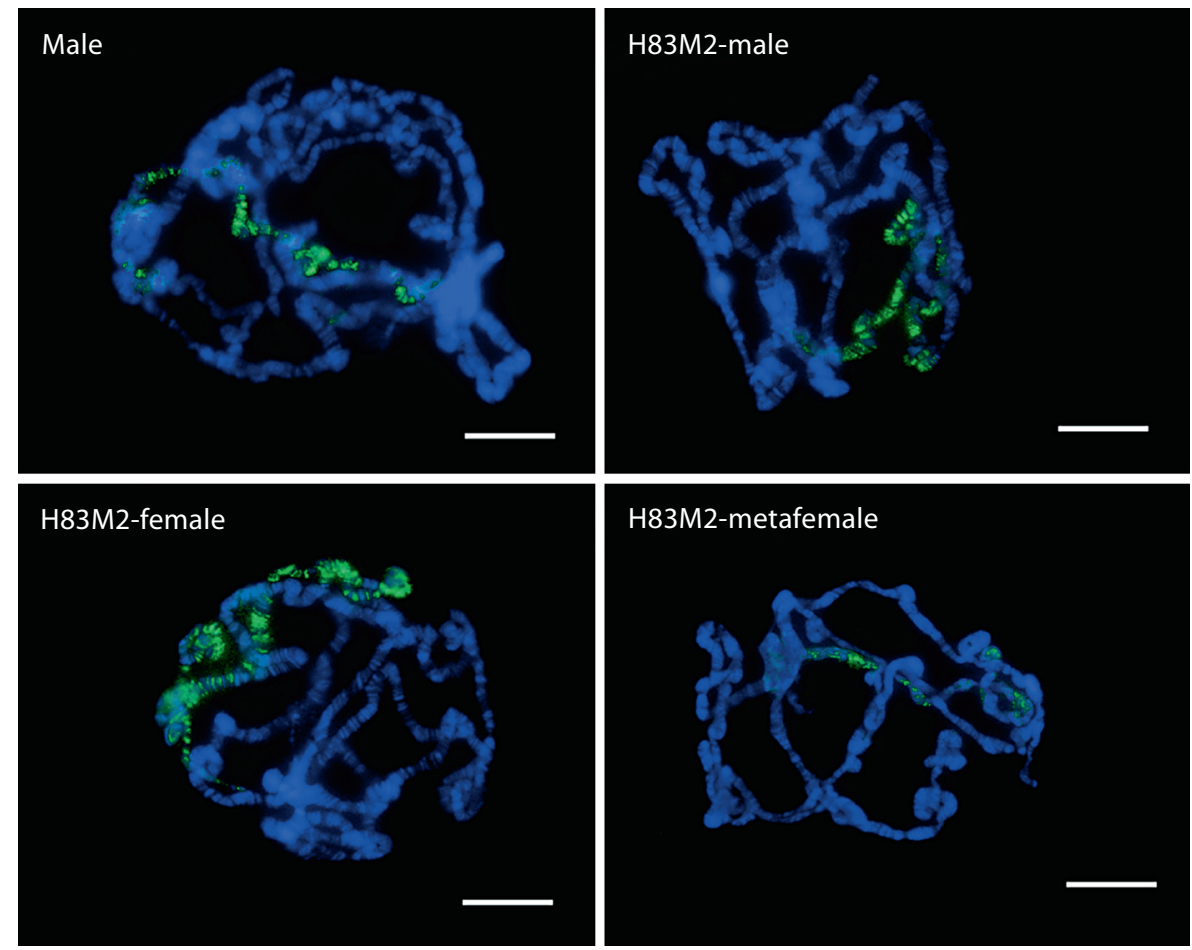

H83M2-metafemale

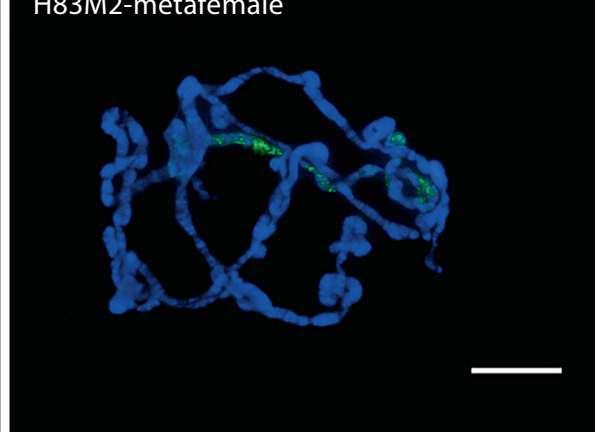

study, we introduced transgenic msl2 into females and metafemales to focus on the effect of MSL2 on MSL sequestration, histone acetylation and gene expression to understand further the function of the complex.

\section{Ectopically Expressed MSL2 Protein Is Present on the X Chromosomes of Transgenic Females and Metafemales}

The expression of MSL2 protein is inhibited in normal females by Sex lethal [Kelley et al., 1995; Zhou et al., 1995; Bashaw and Baker, 1997]. We introduced the msl2 gene, $(w+) H 83 M 2-6 I$ (referred to subsequently as H83M2), in a P-element construct to normal females and metafemales. To confirm the presence of MSL2 in these transgenic flies, immunostaining of polytene chromosomes with MSL2 antibody was carried out among males, females and metafemales with or without the H83M2 transgene (the 6 genotypes are described in Materials and Methods). The results show that MSL2 is present on the X chromosomes of transgenic msl2 females and metafemales (referred to as H83M2-females and H83M2-metafemales, respectively), similarly to normal or overexpressing MSL2 males (referred to as H83M2-males) (fig. 3), while on normal female or metafemale chromosomes, no MSL2 binding was detected (data not shown).
MOF antigen was detected along the $\mathrm{X}$ chromosomes of the H83M2-females and H83M2-metafemales (online supplementary fig. 1; for supplementary materials, see www.karger.com/doi000218134), which was similar to normal and H83M2-males. In order to compare the distribution in normal males, normal females and metafemales, the salivary glands of normal males plus normal females or metafemales were mixed on the same slide,

Fig. 4. Enrichment of H4Ac16 on the X chromosomes of ectopically expressing MSL2 females and metafemales. Immunofluoresence staining of larval polytene chromosomes with antiH4Ac16 (green) from 6 larval genotypes of males, females and metafemales with and without the transgene $(w+) H 83 M 2-6 I$. Blue is DAPI stain for DNA. The left most column shows merged images from the DAPI and anti-H4Ac16 staining. Arrowheads indicate that $\mathrm{H} 4 \mathrm{Ac} 16$ is enriched on the $\mathrm{X}$ chromosomes. The labels on the left side indicate the 6 different genotypes: ( $(+)$ for normal females and (H83M2- + ) for ectopically expressed MSL2 females; ( $₫)$ for normal males and (H83M2- $\delta$ ) for overexpressing MSL2 males; $\left(\mathrm{m}^{\circ}\right)$ for normal metafemales and $\left(\mathrm{H} 83 \mathrm{M} 2-\mathrm{m}^{\circ}\right)$ for ectopically expressing MSL2 metafemales. Normal males, females and metafemales were used as controls. The scale bar represents $15 \mu \mathrm{m}$. 


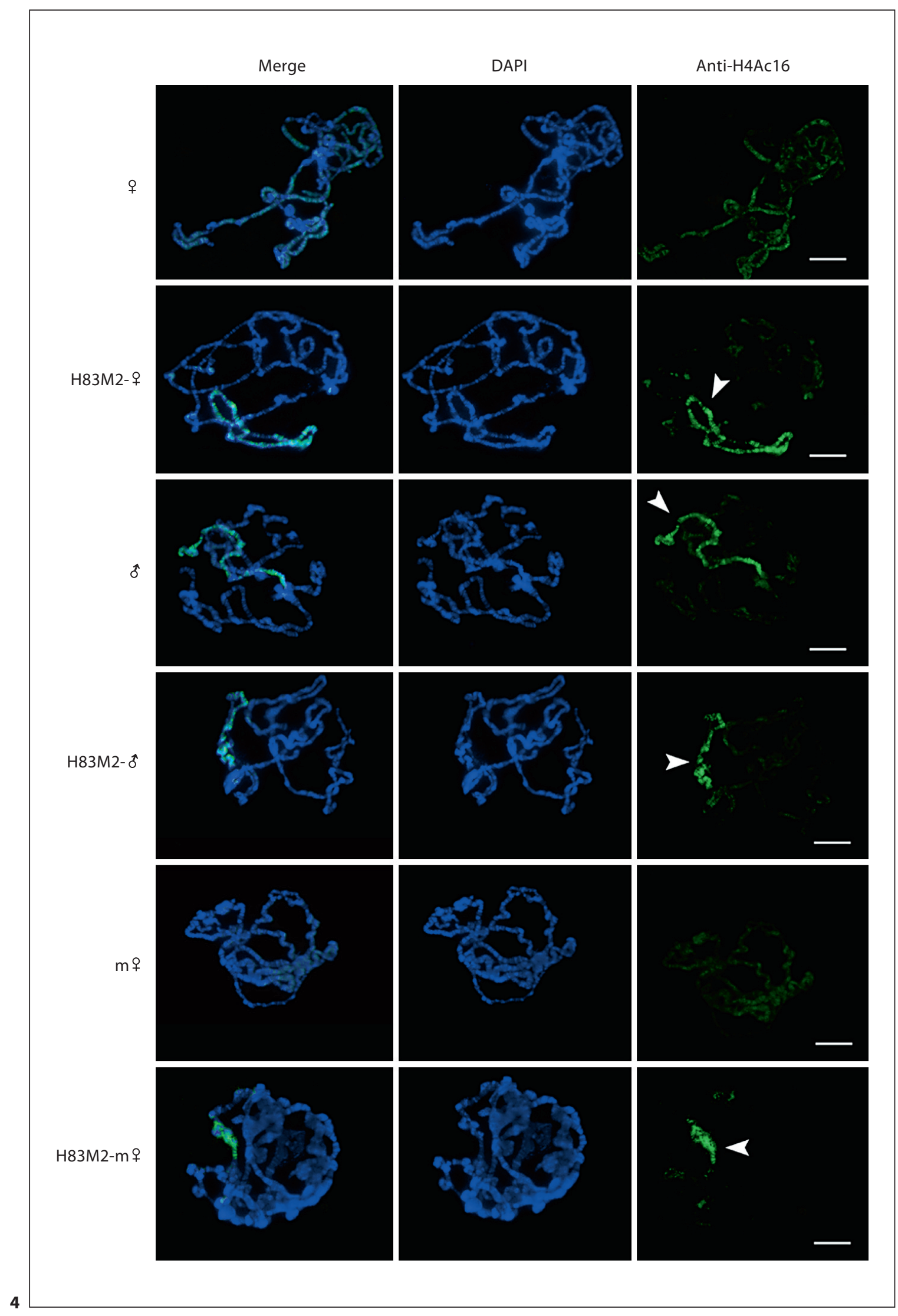


Fig. 5. Effects of ectopically expressing MSL2 on the expression of the X chromosome genes in larvae. The top panel image represents gene transcripts detected by Northern analysis from 6 genotypes of males, females and metafemales with and without $(w+) H 83 M 2-6 I$. The bottom panel is rRNA as a loading control. Each lane was loaded with $10 \mu \mathrm{g}$ of total RNA. The intensity of bands was measured by a Fujifilm Fluorescent Image Analyzer FLA-2000 and analyzed by Fujifilm Image Gauge V 3.3 program (Fuji, Tokyo, Japan). The relative transcript (transcripts/rRNA) levels in the 6 genotypes are presented in the bar graph. The labels of H83M2-male, H83M2-female and H83M2-metafemales indicate the transgenic MSL2 males, females, and metafemale, respectively. No significant differences between the normal and transgenic genotypes are observed in males, females, or metafemales. The metafemale value denoted with a triangle is significantly less than the normal female.

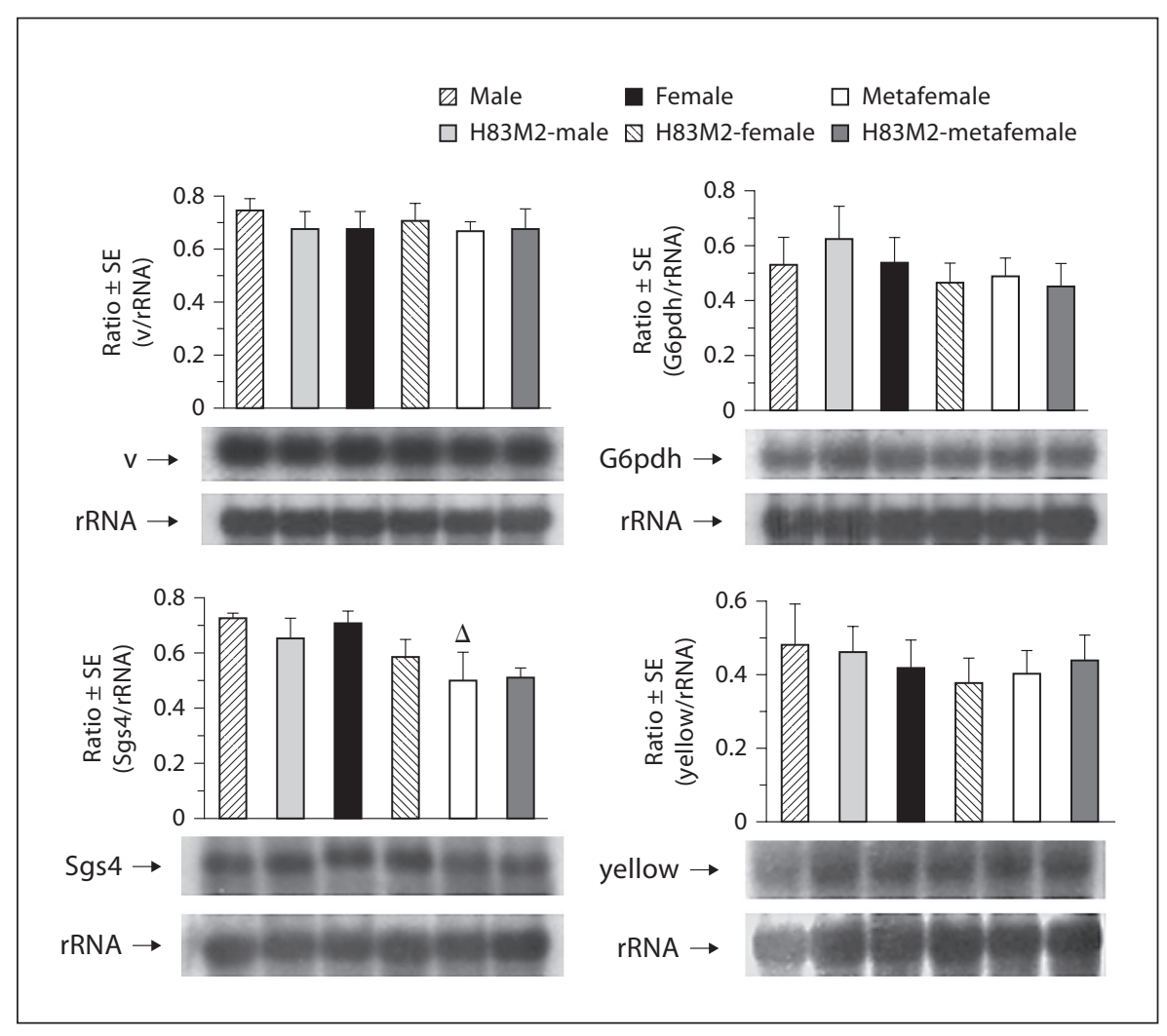

squashed, then double-probed with SXL and MOF antibodies. SXL antibody was used to identify females from males because SXL is female specific [Cline, 1993; Bopp et al., 1996]. The mixture allows direct comparisons of genotypes under identical conditions of fixation and image capture. The results are shown in online suppl. figure 2. Double labeling with MSL2 and MOF antibodies reveals that MOF co-localizes with MSL2 in H83M2 females (online suppl. fig. 3), as previously reported [Gu et al., 1998]. These results confirm that ectopic expression of MSL2 in females and metafemales recruits MOF to the $\mathrm{X}$ chromosome in these experiments.

The distribution of H4Ac16 in polytene chromosomes of normal male, female and metafemales with and without ectopically expressed MSL2 was also examined. The results reveal that the increase of MOF on the X parallels H4LysAc16 enrichment in H83M2-females and H83M2metafemales (fig. 4) although it is important to note that modified H4 is never completely depleted on the autosomes including in normal males. In the normal females and metafemales, the H4Ac16 is detected at a low level and is evenly distributed over the genome compared to autosomal levels in males (fig. 4; online suppl. fig. 4).
These studies establish the chromatin parameters for interpretation of gene expression analyses.

\section{Direct Targeting of MSL2 and MOF on the}

$X$ Chromosomes Does Not Change the X-linked Gene

Expression in Transgenic Females and Metafemales

As shown in the previous sections, the ectopic expression of MSL2 in both females and metafemales sequesters the MSL complex protein MOF to the X from the autosomes, leading to the increase of histone acetylation on the X chromosomes. In order to investigate the effect of this ectopic MSL2 on gene expression, transcript levels were examined in females and metafemales with and without ectopically expressed MSL2, as well as in normal and over-expressing MSL2 males. A Northern analysis was performed to detect mRNA of each gene with ribosomal RNA serving as a gel-loading control. The results show that for diverse selected X-linked genes, including yellow, vermilion, Sgs4 and G6pdh, expression remains unchanged (at the 95\% confidence level) in H83M2-females and -metafemales (fig. 5), relative to respective normal females and metafemales in the same segregating population. Using RNA from the more abundant geno- 
Fig. 6. Effects of ectopically expressing MSL2 on expression of autosomal genes in larvae. Six genotypes of males, females and metafemales with and without transgene $(w+) H 83 M 2-6 I$ were examined for mRNA expression of autosomal genes by Northern analysis. Detailed descriptions are in figure 5. The asterisk denotes a value that is significantly different for each comparison of genotypes with and without H83M2 in males, females or metafemales at the $95 \%\left(^{*}\right)$ or $99 \%\left({ }^{* *}\right)$ confidence level. The metafemale values denoted with a triangle are significantly different from those of the normal female.

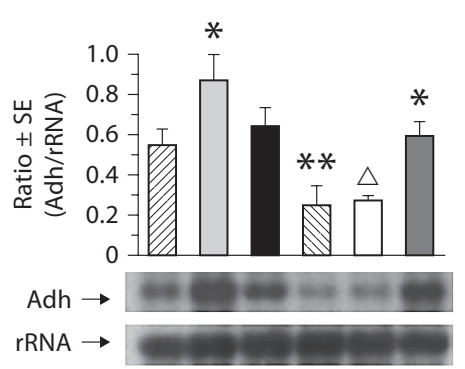

$\square$ Male

$\square$ H83M2-male

- Female

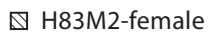

$\square$ Metafemale

$\square$ H83M2-metafemale

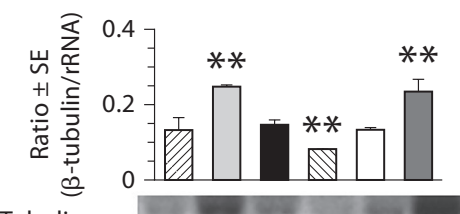

$\beta$-Tubulin $\rightarrow$

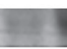

$\mathrm{rRNA} \rightarrow$
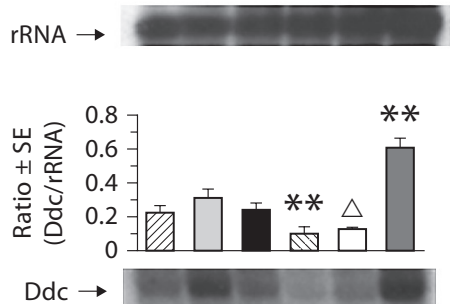

rRNA $\rightarrow$
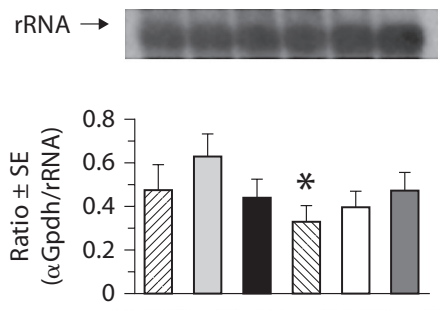

$\alpha \mathrm{Gpdh} \rightarrow$

rRNA $\rightarrow$

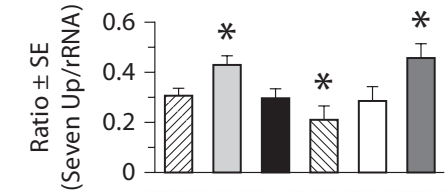

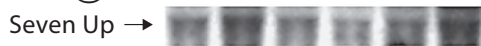

rRNA $\rightarrow$

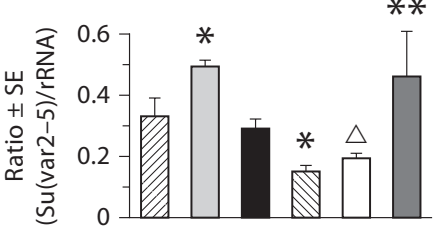

Su(var 2-5) $\rightarrow$

rRNA $\rightarrow$

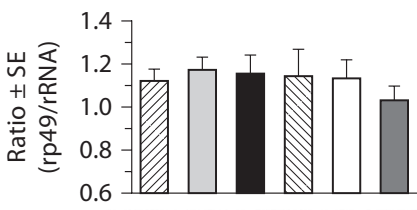

rp49 $\rightarrow$

$\mathrm{rRNA} \rightarrow$ types, the X-linked genes, $6 P g d h$ and rudimentary, were also examined with the result of no increase in ectopically expressing genotypes (online suppl. fig. 5).

\section{Dosage Compensation of X-linked Genes in Metafemales}

When examining gene expression in metafemales (fig. 5; online suppl. fig. 5), we found all examined Xlinked genes are compensated compared to normal females although Sgs 4 is significantly below the female level possibly representing a case of overcompensation. This finding agrees with the results reported by others [Stern, 1960; Lucchesi et al., 1974; Birchler et al., 1989; Birchler, 1992], suggesting that the $3 \mathrm{X}$ chromosomes in metafe- males exhibit dosage compensation having similar total expression to the $2 \mathrm{X}$ s in normal females. In contrast, several autosomal genes had reduced expression in metafemales compared to normal females (fig. 6).

Direct Targeting of MSL2 and MOF to the

$X$ Chromosomes Affects Autosomal Gene Expression

in Transgenic Females

We examined the expression of diverse selected autosomal genes, including $\alpha$-Gpdh, Adh, Suvar(2-5), $\beta$-Tubulin, SevenUp, Rp49 and $D d c$ for the same genotypes of larvae that were noted above. The only autosomal gene that remains unchanged in expression for all genotypes is $r p 49$ (fig. 6). 


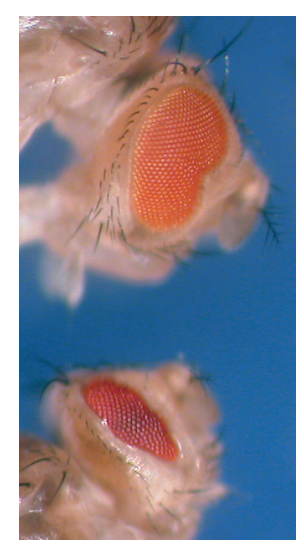

Fig. 7. Phenotypic effect of H83M2 on Bar and mini-white. The top image shows a $\mathrm{Basc} / \mathrm{w}$ - female with the H83M2 transgene, showing the kidney shaped eye typical of a $B /+$ female with no dosage compensation. The bottom shows the typical dosage compensated $B / Y$ male.

\section{Phenotypic Effect of Ectopic MSL2}

To examine the impact of ectopically expressed MSL2 on the phenotypic level, males carrying the H83M2 transgene heterozygous with TM3, Ser were crossed to Basc females. The Basc X chromosome carries the B eye mutation, which is dominant and which exhibits dosage compensation. If the targeting of the MSL complex were to cause Bar to be doubled in expression in response to ectopic dosage compensation, the eye shape would be converted to the narrow Bar phenotype typical of males rather than the kidney shape typical of a $B /+$ female. The latter phenotype was found (fig. 7). Moreover, because Basc carries white-apricot, the expression of the mini-white marker on the autosomal insertion could be monitored. Its expression is reduced in parallel with other autosomal genes.

Moreover, when $C(1) D X, y^{1} w^{1} f^{1}$ attached $\mathrm{X}$ females are crossed by $w-; T M 3 / H 83 M 2$, the resulting progeny have the mini-white on the autosomal transgene and can be scored in males and females. The eye color of females in this case is lighter than the males as is usually the case and thus shows no evidence of acquisition of dosage compensation, which would be indicated by equivalent male and female expression. Furthermore, in a $w$-; msl1/CyO; H83M2-6I/TM3 stock, the $m s l 1 / m s l 1$ females have a darker eye color than do the msll/CyO females, which have the MSL complex sequestered from the autosomes. Thus, elimination of the sequestration of MOF to the X chromosome in the msll/msll genotype also eliminates the effect on mini-white. These phenotypic data are consistent with the molecular findings.

Another dominant mutation on the $\mathrm{X}$ chromosome that exhibits dosage compensation is Beadex of which alleles $B x^{1}$ or $B x^{2}$ show a nicked wing phenotype in hemizygous males that is equivalent to homozygous females.
Heterozygous females show no wing nicking. Females heterozygous for $B x^{1} /+$ and $B x^{2} /+$ on the $\mathrm{X}$ together with the H83M2 transgene show no evidence of acquiring dosage compensation giving a normal wild type wing phenotype in both cases (data not shown). Acquisition of dosage compensation should produce heterozygous $B x /+$ females with nicked wings.

\section{Expression of MSL2 in Metafemales Reverses the}

$X$ Dosage Effect

In H83M2-metafemales, interestingly, the autosomal gene expression is significantly increased compared to regular metafemales for 5 out of 7 genes investigated (fig. 6). In other words, in this case the negative dosage effect of the $3 \mathrm{X}$ chromosomes is ameliorated with a return of expression levels to those similar to normal females. We also examined the autosomal genes in H83M2males. For some genes, such as Adh, $\beta$-Tubulin, SevenUp and $S u(v a r 2-5)$, the expression was significantly increased compared to the normal males (fig. 6).

\section{Discussion}

In this study, the effects of ectopic expression of MSL2 on histone acetylation and gene expression were examined to investigate the function of the MSL complex. The results show that the presence of MSL2 in ectopically expressing MSL2 females and MSL2 metafemales sequesters the MSL proteins to the Xs, which consequently accumulate histone acetylation (fig. 4; online suppl. fig. 1). However, the increased histone acetylation does not change the dosage compensation behavior of X-linked gene transcripts or phenotypes in H83M2-females and H83M2-metafemales (fig. 5). Thus, the sampled X-linked genes show little response in gene expression to the introduction of the transgene H83M2. The function of the MSL complex on the X chromosome(s) appears to inhibit the response of genes to high levels of histone acetylation brought about by the complex. The mechanism of compensation must differ from one in which the MSL complex directly mediates dosage compensation of the $\mathrm{X}$ via histone acetylation levels [Belote and Lucchesi, 1980; Kuroda et al., 1991; Bone et al., 1994; Jin et al., 1999]. Our results are consistent with the hypothesis that some component of the MSL complex or associated activities counteracts the impact of the observed high levels of histone acetylation. Some potential candidates that might act to produce this effect include the ISWI chromatin remodeling factor and the HP1 complex [Bhadra et al., 1999; de 
Wit et al., 2005; Pal Bhadra et al., 2005; Spierer et al., 2005].

Previous results on ISWI mutants indicate that it is required for the override of the histone acetylation $[\mathrm{Pal}$ Bhadra et al., 2005]. This proposal is suggested because the ISWI mutant shows a 'bloated' X chromosome and an increase of X-linked gene expression in males. This chromosomal phenotype is modulated by the amount of H4Ac16 [Corona et al., 2002]. Recent studies show that the repressive Heterochromatin Protein 1 (HP1) complex is localized on the male $\mathrm{X}$ and might also act to counteract the effect of histone acetylation [de Wit et al., 2005; Spierer et al., 2005], although this proposition has not been directly tested.

Our previous results studying gene expression in embryos [Pal Bhadra et al., 2005] indicated that mutation of mle did not eliminate dosage compensation of most $\mathrm{X}$-linked genes but did result in expression increases for autosomal loci. Gene expression analyses of Sex lethal (Sxl) mutations found no increase in expression for $\mathrm{X}$-linked genes but reductions for the autosomes [PalBhadra et al., 2005]. This genotype would allow expression of MSL2 and cause MSL complex formation on the $\mathrm{X}$ chromosomes [Bhadra et al., 2000]. The results with ISWI mutant embryos are noted above. The double mutant $m o f^{l}$; mle embryos showed a loss of dosage compensation. This genotype shows lethality in embryonic stages in contrast to the other genotypes, which do not affect dosage compensation and which survive to larval stages. When comparing the results at the embryonic and larval stages, the generalized trends of $\mathrm{X}$ and autosomal gene expression in any one mutation are quite similar [Bhadra et al., 2000; Pal Bhadra et al., 2005], suggesting attrition during development accounts for the reduced survival of the $m s l$ and $S x l$ mutants rather than a fundamental difference in gene expression between embryos and larvae. The current study was conducted using larvae in order to classify metafemales using the $y+$ gene marker. The differential survival of the various mutant genotypes compared to normal is likely contributed to by the modulations of autosomal expression, none of which are of the magnitude found with loss of compensation and thus is consistent with their survival into the larval stages. Our results demonstrate that metafemales that survive to larval stages do not acquire the MSL complex on their Xs as the mechanism of dosage compensation and indeed a down regulation is needed to explain compensation in this genotype.

Our results with dosage compensation in metafemales are consistent with previous findings of little to no change of total X-linked gene expression compared to normal females [Margolis, 1934; Stern, 1960; Lucchesi et al., 1974; Birchler et al., 1989; Birchler, 1992]. The immunolocalization studies demonstrate that no MSL complex is present on the $3 \mathrm{X}$ chromosomes in metafemales, thus indicating that compensation in this genotype can not be mediated by the MSL complex. Also, our current results extend the finding that many autosomal genes have reduced expression in metafemales [Birchler et al., 1989, 1992]. Previous results have suggested a mechanistic relationship between male and metafemale compensation [Birchler, 1992]. We note that compensation of linked genes on the trisomic chromosome and reductions in expression of numerous unlinked genes is analogous to the situation with large autosomal trisomies in which autosomal dosage compensation occurs as well as an inverse down-regulation effect on many genes encoded elsewhere in the genome [Devlin et al., 1982, 1988; Birchler et al., 1990].

In H83M2-females and H83M2-metafemales, the $\mathrm{H} 4 \mathrm{Ac} 16$ on the autosomes is strongly depleted due to the sequestration of MSL complex to the Xs (fig. 4; online suppl. fig. 1). The reduced H4Ac16 on the autosomes accompanies lower autosomal gene expression in H83M2females, but higher expression in metafemales, compared to their respective control (fig. 6). In general, gene expression on the autosomes responds positively to the level of histone acetylation, which was found in the present and previous studies of H83M2-females [Bhadra et al., 1999, 2000; Pal Bhadra et al., 2005]. However, in contrast to the H83M2-females, the H83M2-metafemales have increased autosomal expression relative to the unmodified metafemale genotype. This effect seems unlikely to be attributed to increased autosomal H4Ac16 because MOF has been sequestered to the $\mathrm{X}$ and removed from the autosomes. One possible interpretation is that histone acetylation depletion eliminates the inverse dosage effect of the $3 \mathrm{Xs}$ in metafemales as has been postulated to occur in normal males [Bhadra et al., 1999]. In that case, it is hypothesized that depletion of H4Ac16 on the autosomes reduces an otherwise generalized up-regulation as suggested by the observation that there is an up-regulation of autosomal genes when the MSL complex is disrupted, thus freeing MOF to a uniform genome-wide distribution and concomitant H4Lys16Ac modification [Bhadra et al., 1999]. However, other possible explanations might account for the modulation of autosomal gene expression in MSL2 expressing metafemales.

The effect of over-expressing MSL2 on autosomal gene expression in transgenic H83M2-males was also ad- 
dressed. There was no change in expression of any Xlinked gene tested. The results reveal that overexpressing MSL2 significantly enhanced the autosomal gene expression for most genes tested (fig. 6), although there was no obvious enhanced signal of histone acetylation in H83M2males compared to the normal males (fig. 4). Whether there is an undetectable reversal of MOF localization, a redistribution of other chromatin modifiers implicated in X and autosomal differences, such as HP1 or JIL1 kinase, or other changes is not known. The nature of the experiment requires comparison of segregating chromosomes. It seems unlikely that this effect is due to segregating general modifiers of gene expression because the effects are specific to the autosomes and to the male sex.

The present data are consistent with the hypothesis that the MSL complex plays a role in overriding the effect of histone acetylation on the male $\mathrm{X}$ as well as in maintaining the autosomal equivalence between the 2 sexes. It is hypothesized that in males ( $1 \mathrm{X}: 2 \mathrm{~A}$ ), the $\mathrm{X}$ is twofold upregulated by the genomic imbalance of regulatory genes to equal the 2 Xs in females [Birchler et al., 2001, 2006]. In metafemales (3X: $2 \mathrm{~A}$ ), each of the $3 \mathrm{X}$ chromosomes is down-regulated by two thirds by the genomic imbalance to equal the $2 \mathrm{Xs}$ in normal females. When the data on triploid intersexes $(2 \mathrm{X} ; 3 \mathrm{~A})$ and triploid metamales (1X; 3A) [see Birchler et al., 2006] are also considered, the expression of the $\mathrm{X}$ is modulated in each case to the inverse ratio of the $\mathrm{X}$ to autosomal ratio (i.e., an inverse relationship between chromosomal dosage and gene expression). The inverse dosage effect of the $\mathrm{X}$ for a twofold up-regulation in normal males might be expected to also exert the same fold up-regulation on the autosomes. This potential up-regulation of the autosomes by chromosomal imbalance might be prevented by the removal of MOF from the autosomes to lower the histone acetylation level so as to maintain equal expression between the sexes.

As noted above, a model prevalent in the literature hypothesizes that the MSL complex and its coincident histone modifications alone bring about the twofold increase in expression of the single $\mathrm{X}$ in males. While this model may be simple, there are several facts that are not consistent with it. Foremost is the finding that dosage compensation for many genes is still present when the MSL complex is destroyed by a variety of means [Hiebert and Birchler, 1994; Bhadra et al., 1999, 2000; Pal Bhadra et al., 2005,2006 and the observation that dosage compensation occurs in the germline where there is no MSL complex [Parisi et al., 2003; Gupta et al., 2006]. Secondly, it does not explain the autosomal increases that are found in various mutants that eliminate the MSL complex.
Thirdly, it can not explain the various levels of modulations of gene expression that are needed to account for dosage compensation in the different doses of the X chromosome other than the normal male as described above, in particular the triple $\mathrm{X}$ metafemales as examined in this study.

A critical issue to determine the role of the male specific lethal genes is the mutant effect on gene expression. The original, now decades old, studies analyzed the autoradiographic grain counts from tritiated uridine incorporated into nascent RNA on polytene chromosomes, as a measure of the rate of transcription. The data were treated as a relative number from the $\mathrm{X}$ chromosome compared to the autosomes [Belote and Lucchesi, 1980; Okuno et al., 1984]. This ratio is diminished to nearly half in males mutant for the maleless (mle) or msl3 genes, whose mutation eliminates the MSL sequestration to the X [Kuroda et al., 1991]. There are 2 ways to achieve such a ratio change in the mutant males. The $\mathrm{X}$ could be reduced in expression due to a loss of dosage compensation and the autosomes are not changed in the mutant relative to normal males. The second way to reduce the ratio is if the $\mathrm{X}$ basically retains dosage compensation and the autosomes are increased in expression. Inspection of the grain count data favors the latter [Belote and Lucchesi, 1980; Okuno et al., 1984]. A survey of $39 \mathrm{X}$ and autosomal genes in mle and normal males with absolute determinations of expression relative to DNA demonstrated that the latter situation was indeed the case [Hiebert and Birchler, 1994]. These results have been confirmed by fluorescent in situ hybridization studies of embryos [Pal Bhadra et al., 2005], which also demonstrate phenotypically the absolute gene expression of the $\mathrm{X}$ and the autosomes when the MSL complex is destroyed in vivo. The compensation of the $\mathrm{X}$ and an approximate doubling of the autosomes found in such mutant males is the predicted effect of the inversely acting dosage dependent regulatory hierarchy when the dosage of the $\mathrm{X}$ is reduced to one copy in males.

In recent studies, Hamada et al. [2005] and Straub et al. [2005] purported to test models of dosage compensation in Drosophila by using RNA interference to eliminate the male specific lethal 2 (MSL2) protein in Drosophila tissue culture cells. These conditions were found to cause the dissociation of the MSL complex and to reduce drastically the levels of histone 4 lysine 16 (H4K16) acetylation in these cells. By various techniques, including Affymetrix chip analysis, gene expression was examined. The authors sought to test an 'activation' model in which the MSL complex brings the MOF histone acety- 
lase to the male $\mathrm{X}$ to cause a twofold up-regulation to achieve dosage compensation. The other model was referred to as the 'inverse' model, which suggests that the genomic imbalance in the male genotype would tend to up-regulate the whole genome by approximately twofold including the autosomes. The MSL complex would sequester the MOF acetylase from the autosomes to mitigate this effect and then the high levels of histone acetylation on the X would be counteracted by the MSL complex and associated activities to prevent overcompensation. However, the MSL complex and associated activities would allow the twofold up-regulation to occur on the $\mathrm{X}$ to achieve the proper level of dosage compensation.

However, to the extent that the MSL complex is dissociated and the genome-wide level of $\mathrm{H} 4 \mathrm{~K} 16$ acetylation is virtually nil [as suggested by Hamada et al., 2005], both the 'activation' and 'inverse' models they sought to test would predict the same results. Indeed, in Pal Bhadra et al. [2005], it was reported that X linked genes lost compensation and there were no autosomal increases in the mof; maleless (mof; mle) double mutants. This genotype would disrupt the MSL complex and result in basically no genomic wide $\mathrm{H} 4 \mathrm{~K} 16$ acetylation in the same manner as claimed for the RNAi experiments. Previous data had led to the suggestion that the MOF histone acetylase was sequestered from the autosomes to prevent their up regulation [Bhadra et al., 1999]; thus, if there is no available acetylation, there could be no autosomal increase. In the segregating genotypes described previously [Hiebert and Birchler, 1994; Bhadra et al., 1999, 2000], the mle mutants generally retained dosage compensation and had autosomal increases. This previous work had also found a genome-wide distribution of H4Lys16 Ac in the mle mutants compared to the chromosomes of normal males present in mixtures on the same slide [Bhadra et al., 1999]. The mle mutation causes a release of the MOF acetylase from the male $\mathrm{X}$, which catalyzes a more uniform acetylation across the genome in vivo. The degree of genome-wide acetylation in the mle mutants appears to be greater than in the RNAi experiments using tissue culture cells reported by Hamada et al. [2005] and Straub et al. [2005].

While having noted the above, it does appear from the data of Hamada et al. [2005] that there might be residual genome-wide acetylation. Indeed, the data indicate that there are autosomal increases. As Hamada et al. note, the data analysis was conducted by correcting the individual gene expression by the average genome-wide expression using Affymetrics software. They correctly note that if autosomal increases were occurring, the $\mathrm{X}$ reductions would appear to be enhanced and the autosomal increases would be diminished in the treated data (because there are roughly 4 times as many autosomal genes as X-linked genes). Indeed, in their data that documented expression changes at a 1.2 cutoff, the up-regulated genes are predominantly skewed to the autosomes and the reductions are predominantly skewed to the X. A chi square analysis of the distribution of up-regulation on the autosomes versus the $\mathrm{X}$ (using a $4 \mathrm{~A}: 1 \mathrm{X}$ random expectation; 1 d.f.) shows that the probability that this partitioning into these classes is due to chance alone is so low as to be virtually impossible. Also, the log2 curves of autosomal gene expression shown are not normally distributed around zero, but are always shifted to higher expression. This is precisely the type of result that would be produced from the data analysis used. There was no attempt to validate the up-regulated autosomal genes, so it is difficult to determine the exact magnitude of these autosomal increases from the analysis. Nevertheless, it seems reasonable that there is a greater loss of dosage compensation in the RNA $\mathrm{i}$ knockdown experiment than occurs with mle in vivo (in which a few X-linked genes do show loss of compensation [Hiebert and Birchler, 1994; Bhadra et al., 1999]) perhaps because of differences in residual histone acetylation. Hamada et al. also note that there was a substantial fraction of X linked genes that retained compensation, but these are likely underestimated by the analysis used; these might also result from residual genome-wide acetylation. The results fall between the extremes of no MSL complex and no genome-wide acetylation, which would result in no compensation and no autosomal changes in both models, versus the situation in which there is no MSL complex with a redistribution of acetylation as occurs in the mle mutants, which exhibits a greater number of compensated genes and autosomal increases.

More recently, in a study of gene expression in larvae mutant for the roX RNAs, Deng and Meller [2007] first treated their data by equating the median values of autosomal genes in mutant and wild type larvae, a situation more parallel to the mle studies, and then normalizing the $\mathrm{X}$ values to the respective autosomes. This analysis basically equates autosomal expression without independent evidence to test the assumption of their equality but in their data, the $\log 2$ ratio plot shows an obvious greater expression of the autosomes. Thus, if an absolute measure were made for autosomal genes, even greater increases would be observed. For the X chromosome, Deng and Meller [2007] found a few genes in the mutants approaching $50 \%$ of the normal level but others were less reduced. Because of the data treatment noted above, the $\mathrm{X}$ reduc- 
tions will be erroneously produced by the shifting downward of a normal $\log 2$ ratio distribution around zero, which represents no change. Thus, these data also support the contention that most $\mathrm{X}$ linked genes retain compensation when the MSL complex is disrupted in vivo and that many autosomal increases occur.

Thus, there does not appear to be a significant conflict between our results that assayed individual genes by Northern quantification standardized to absolute DNA values or by phenotypic analysis in embryos and the global analyses discussed above. Therefore, the MSL complex alone can not be responsible for the twofold upregulation of the male $\mathrm{X}$ chromosome but requires an appropriate dosage dependent component in the magnitude of an inverse effect. The results presented in this paper showing that ectopic targeting of the MSL complex does not cause a twofold upregulation of the $\mathrm{X}$ chromosomes is consistent with this view.

An inverse dosage effect occurs in most aneuploids of substantial length in diverse multicellular eukatyotes as noted in the introduction. Thus, it likely provides the dosage component of both the dosage compensation of the male $\mathrm{X}$ and of the triple $\mathrm{X}$ genotype in metafemales.
The apparent role of the MSL complex appears to be to ameliorate the inverse effect that might otherwise occur for the autosomes by sequestering MOF to the X chromosome in males and then to counteract the high level of histone acetylation that results to prevent over compensation but at the same time allowing the proper twofold upregulation to occur to achieve dosage compensation [Bhadra et al., 1999; Pal Bhadra et al., 2005]. This model proposes a single mechanism that explains the multiple levels of gene expression in different genotypes exhibiting dosage compensation, the MSL binding on the X in males, the expression patterns in the sex specific lethal mutations as well as dosage compensation in metafemales [Birchler, 1996].

\section{Acknowledgements}

We thank Dr. M. Kuroda for providing MSL2 and MOF antibodies, and Drs. N. Riddle and S. Elgin for Su(var)2-5 cDNA. We thank an anonymous reviewer for suggesting a comparison of models of dosage compensation. This work was supported by NIH grant R01 JM068042.

\section{References}

Ait Yahya-Graison E, Aubert J, Dauphinot L, Rivals I, Prier M, et al: Classification of human chromosome 21 over-expression variations in Down syndrome: impact on disease phenotypes. Am J Hum Genet 81:475-491 (2007).

-Altug-Teber O, Bonin M, Walter M, Mau-Holzman UA, Dufke A, et al: Specific transcriptional changes in human fetuses with autosomal trisomies. Cytogenet Genome Res 119: 171-184 (2007).

- Bashaw GJ, Baker BS: The regulation of the Drosophila msl-2 gene reveals a function for Sexlethal in translational control. Cell 89:789798 (1997).

Belote JM, Lucchesi JC: Control of X chromosome transcription by the maleless gene in Drosophila. Nature 285:573-575 (1980).

-Bhadra U, Pal-Bhadra M, Birchler JA: Role of the male specific lethal $(\mathrm{msl})$ genes in modifying the effects of sex chromosomal dosage in Drosophila. Genetics 152:249-268 (1999).

- Bhadra U, Pal-Bhadra M, Birchler JA: Histone acetylation and gene expression analysis of Sex lethal mutants in Drosophila. Genetics 155:753-763 (2000).

-Birchler JA: A study of enzyme activities in a dosage series of the long arm of chromosome one in maize. Genetics 92:1211-1229 (1979).
Birchler JA: The genetic basis of dosage compensation of alcohol dehydrogenase-1 in maize. Genetics 97:625-637 (1981).

- Birchler JA: Expression of cis-regulatory mutations of the white locus in metafemales of Drosophila melanogaster. Genet Res 59:1118 (1992).

Birchler JA: X chromosome dosage compensation in Drosophila. Science 272:1190 (1996).

Birchler JA, Newton KJ: Modulation of protein levels in chromosomal dosage series of maize: The biochemical basis of aneuploid syndromes. Genetics 99:247-266 (1981).

Birchler JA, Veitia RA: The gene balance hypothesis: From classical genetics to modern genomics. Plant Cell 19:395-402 (2007).

-Birchler JA, Hiebert JC, Krietzman M: Gene expression in adult metafemales of Drosophila melanogaster. Genetics 122:869-879 (1989).

Birchler JA, Hiebert JC, Paigen K: Analysis of autosomal dosage compensation involving the alcohol dehydrogenase locus in Drosophila melanogaster. Genetics 124:677-686 (1990).

- Birchler JA, Bhadra U, Pal Bhadra M, Auger DL: Dosage-dependent gene regulation in multicellular eukaryotes: implications for dosage compensation, aneuploid syndromes and quantitative traits. Dev Biol 234:275-288 (2001).
Birchler JA, Riddle NC, Auger DL, Veitia RA: Dosage balance in gene regulation: biological implications. Trends Genet 21:219-226 (2005).

- Birchler JA, Fernandez HR, Kavi HH: Commonalities in compensation. Bioessays 28 : 565-568 (2006).

Bone JR, Lavender J, Richman R, Palmer MJ, Turner BM, et al: Acetylated histone $\mathrm{H} 4$ on the male $\mathrm{X}$ chromosome is associated with dosage compensation in Drosophila. Genes Dev 8:96-104 (1994).

Bopp D, Calhoun G, Horabin JI, Samuels M, Schedl P: Sex-specific control of Sex-lethal is a conserved mechanism for sex determination in the genus Drosophila. Development 122:971-982 (1996).

Brownell JE, Allis CD: Special HATs for special occasions: linking histone acetylation to chromatin assembly and gene activation. Curr Opin Genet Dev 6:176-184 (1996).

Cline TW: The Drosophila sex determination signal: how do flies count to two? Trends Genet 9:385-90 (1993).

-Corona DF, Clapier CR, Becker PB, Tamkun JW: Modulation of ISWI function by site-specific histone acetylation. EMBO Rep 3:242-247 (2002). 
Deng X, Meller VH: roX RNAs are required for increased expression of $\mathrm{X}$-linked genes in Drosophila melanogaster males. Genetics 174:1859-1866 (2007)

Devlin RH, Holm DG, Grigliatti TA: Autosomal dosage compensation in Drosophila melanogaster strains trisomic for the left arm of chromosome 2. Proc Natl Acad Sci USA 79: 1200-1204 (1982).

-Devlin RH, Holm DG, Grigliatti TA: The influence of whole-arm trisomy on gene expression in Drosophila. Genetics 118:87-101 (1988).

-de Wit E, Greil F, van Steensel B: Genome-wide HP1 binding in Drosophila: developmental plasticity and genomic targeting signals. Genome Res 15:1265-1273 (2005).

Grunstein M: Histone acetylation in chromatin structure and transcription. Nature 389: 349-352 (1997).

- Gu W, Szauter P, Lucchesi JC: Targeting of MOF, a putative histone acetyl transferase, to the $\mathrm{X}$ chromosome of Drosophila melanogaster. Dev Genet 22:56-64 (1998).

- Guo M, Birchler JA: Trans-acting dosage effects on the expression of model gene systems in maize aneuploids. Science 266:1999-2002 (1994).

- Guo M, Davis D, Birchler JA: Dosage effects on gene expression in a maize ploidy series. Genetics 142:1349-1355 (1996).

-Gupta V, Parisi M, Sturgill D, Nuttall R, Doctolero M, et al: Global analysis of X-chromosome dosage compensation. J Biol 5:3 (2006).

- Hamada FN, Park PJ, Gordadze PR, Kuroda MI: Global regulation of $\mathrm{X}$ chromosomal genes by the MSL complex in Drosophila melanogaster. Genes Dev 19:2289-2294 (2005).

-Hiebert JC, Birchler JA: Effects of the maleless mutation on $\mathrm{X}$ and autosomal gene expression in Drosophila melanogaster. Genetics 136:913-926 (1994).

- Hilfiker A, Yang Y, Hayes DH, Beard CA, Manning JE, et al: Dosage compensation in Drosophila: the X-chromosomal binding of MSL- 1 and MLE is dependent on Sxl activity. EMBO J 13:3542-3550 (1994).

-Hilfiker A, Hilfiker-Kleiner D, Pannuti A, Lucchesi JC: mof, a putative acetyl transferase gene related to the Tip60 and MOZ human genes and to the SAS genes of yeast, is required for dosage compensation in Drosophila. EMBO J 16:2054-2060 (1997).
Jin Y, Wang Y, Walker DL, Dong H, Conley C, et al: JIL-1: a novel chromosomal tandem kinase implicated in transcriptional regulation in Drosophila. Mol Cell 4:129-135 (1999).

Jin Y, Wang Y, Johansen J, Johansen KM: JIL-1, a chromosomal kinase implicated in regulation of chromatin structure, associates with the male specific lethal (MSL) dosage compensation complex. J Cell Biol 149:1005-1010 (2000).

Kelley RL, Solovyeva I, Lyman LM, Richman R, Solovyev V, et al: Expression of msl-2 causes assembly of dosage compensation regulators on the $\mathrm{X}$ chromosomes and female lethality in Drosophila. Cell 81:867-877 (1995).

Kuroda MI, Kernan MJ, Kreber R, Ganetzky B, Baker BS: The maleless protein associates with the $\mathrm{X}$ chromosome to regulate dosage compensation in Drosophila. Cell 66:935947 (1991).

Lucchesi JC, Rawls JM, Maroni G: Gene dosage compensation in metafemales (3X:2A) of Drosophila. Nature 248:564-567 (1974).

Margolis OS: The effect of a supernumerary X chromosome on members of the Bar series of Drosophila. Genetics 19:118-184 (1934).

- Meller VH, Wu KH, Roman G, Kuroda MI, Davis RL: roX1 RNA paints the $\mathrm{X}$ chromosome of male Drosophila and is regulated by the dosage compensation system. Cell 88:445457 (1997).

Muller H: Further studies on the nature and causes of gene mutations. Proc 6th Int Congr Genet 1:213-255 (1932).

Nguyen DK, Disteche CM: Dosage compensation of the active $\mathrm{X}$ chromosome in mammals. Nat Genet 38:47-53 (2006).

Okuno T, Satou T, Oishi K: Studies on the sexspecific lethals of Drosophila melanogaster. VII. Sex-specific lethals that do not affect dosage compensation. Jpn J Genet 59:237247 (1984).

Pal Bhadra M, Bhadra U, Birchler JA: Cosuppression in Drosophila: gene silencing of $\mathrm{Al}$ cohol dehydrogenase by white-Adh transgenes is Polycomb dependent. Cell 90:479490 (1997).

- Pal Bhadra M, Bhadra U, Kundu J, Birchler JA: Gene expression analysis of the function of the male-specific lethal complex in Drosophila. Genetics 169:2061-2074 (2005).
Pal Bhadra M, Bhadra U, Birchler JA: Misregulation of Sex-lethal and disruption of MSL complex localization in Drosophila species hybrids. Genetics 174:1151-1159 (2006).

- Parisi M, Nutell R, Naiman D, Bouffard G, Malley J, et al: Paucity of genes on the Drosophila $\mathrm{X}$ chromosome showing male-biased expression. Science 299:697-671 (2003).

Rastelli L, Kuroda MI: An analysis of maleless and histone $\mathrm{H} 4$ acetylation in Drosophila melanogaster spermatogenesis. Mech Dev 71:107-117 (1998).

Sambrook J, Russell DW: Molecular Cloning, Third Ed, Appendix 1 (Cold Spring Harbor Laboratory Press, Cold Spring Harbor 2001).

- Spierer A, Seum C, Delattre M, Spierer P: Loss of the modifiers of variegation $\mathrm{Su}(\mathrm{var}) 3-7$ or HP1 impacts male X polytene chromosome morphology and dosage compensation. J Cell Sci 118:5047-5057 (2005).

Stern C: Dosage compensation-development of a concept and new facts. Can J Genet Cytol 2: 105-118 (1960).

Straub T, Gifillan GD, Maier VK, Becker PB: The Drosophila MSL complex activates the transcription of target genes. Genes Dev 19: 2284-2288 (2005).

Turner BM, Birley AJ, Lavender J: Histone H4 isoforms acetylated at specific lysine residues define individual chromosomes and chromatin domains in Drosophila polytene nuclei. Cell 69:375-384 (1992).

-Veitia RA, Bottani S, Birchler JA: Cellular reactions to gene dosage imbalance: genomic, transcriptomic and proteomic effects. Trends Genet 24:390-397 (2008).

-Wang Y, Zhang W, Jin Y, Johansen J, Johansen KM: The JIL-1 tandem kinase mediates histone $\mathrm{H} 3$ phosphorylation and is required for maintenance of chromatin structure in Drosophila. Cell 105:433-443 (2001).

-Wolffe AP, Pruss D: Targeting chromatin disruption: Transcription regulators that acetylate histones. Cell 84:817-819 (1996).

Zhou S, Yang Y, Scott MJ, Pannutti A, Fehr KC, et al: Male-specific lethal 2, a dosage compensation gene of Drosophila, undergoes sex-specific regulation and encodes a protein with a RING finger and a metallothioneinlike cysteine cluster. EMBO J 14:2884-2895 (1995). 